\title{
AN INDOOR NAVIGATION APPROACH CONSIDERING OBSTACLES AND SPACE SUBDIVISION OF 2D PLAN
}

\author{
Man Xu ${ }^{\text {a, b }}$, Shuangfeng Wei ${ }^{\text {a, b, c, } * \text {, Sisi Zlatanova }}{ }^{c}$ \\ ${ }^{a}$ School of Geomatics and Urban Spatial Information, Beijing University of Civil Engineering and Architecture, Beijing, \\ China-1554237194@qq.com, weishuangfeng@ bucea.edu.cn \\ ${ }^{\mathrm{b}}$ Key Laboratory for Urban Geomatics of National Administration of Surveying, Mapping and Geoinformation, \\ Beijing, China - 1554237194@qq.com, weishuangfeng@bucea.edu.cn \\ c 3D Geoinformation, Department of Urbanism, Faculty of Architecture and The Built Environment, Delft University of \\ Technology, Delft, The Netherlands - S.Wei-1@tudelft.nl, S.Zlatanova@tudelft.nl
}

Commission IV, WG IV/7

KEYWORDS: Indoor Navigation, Obstacles, Space Subdivision, Navigation Network, Shortest Path, 2D Plan

\begin{abstract}
:
The demand for indoor navigation is increasingly urgent in many applications such as safe management of underground spaces or location services in complex indoor environment, e.g. shopping centres, airports, museums, underground parking lot and hospitals. Indoor navigation is still a challenging research field, as currently applied indoor navigation algorithms commonly ignore important environmental and human factors and therefore do not provide precise navigation. Flexible and detailed networks representing the connectivity of spaces and considering indoor objects such as furniture are very important to a precise navigation. In this paper we concentrate on indoor navigation considering obstacles represented as polygons. We introduce a specific space subdivision based on a simplified floor plan to build the indoor navigation network. The experiments demonstrate that we are able to navigate around the obstacles using the proposed network. Considering to well-known path-finding approaches based on Medial Axis Transform (MAT) or Visibility Graph (VG), the approach in this paper provides a quick subdivision of space and routes, which are compatible with the results of VG.
\end{abstract}

\section{INTRODUCTION}

With the increased urbanization, public buildings such as shopping centres, airports, museums and music halls have become larger and more complex. Therefore it becomes difficult for people to arrive at a specific room or place even if they are familiar with the environment (Mortari et al., 2014). Indoor navigation is still a challenging research field for several reasons: positioning is not very accurate, users can move freely inside, path network construction process may not be easy and straightforward due to the indoor space complex configurations

\footnotetext{
* Corresponding author
}

(Worboys, 2011; Mortari et al., 2014; Zlatanova et al 2014). Therefore, indoor navigation attracts the attention of a large number of researchers.

Various topics are investigated for indoor navigation, but indoor space modelling is considered to be the key issue to provide an appropriate path for navigation. Much research has been completed on developing semantically-rich models to provide automatic derivation of a navigation network. Li and Lee (2008) build a topological semantic location model based on an exit-location matrix, which reveals semantic relationships and semantic distance of indoor environment. Topological 
characteristics such as connectivity and inclusion are defined to n-ary relationships among entities. Then the Q-analysis method is used to investigate the semantic information of the model $(\mathrm{Li}$ and Lee, 2008). Zhao and Liu (2010) propose an algorithm based on position-export model and formal concept analysis theory. In this model, indoor environment is represented as concept lattice. Making use of neighbouring relationships, A* algorithm is used to find the optimal path between two entities. Liu and Zlatanova (2011) propose the 'door-to-door' approach based on Visibility Graph (De Berg et al., 2000), which derives the connections between doors, which are used to create the network for navigation. This network adapts better to the walking behaviour of pedestrians. This approach takes into account navigation with obstacles (Liu and Zlatanova, 2015). Goetz and Zipf (2011) also use 'door-to-door' approach to create a navigation network for path-finding. They represent the indoor environment with a model which contains topologic, semantic and geometric information. Vertical parts such as elevators and stairs are taken into account with given weights. Then an optimal route between two points is computed with the weights. Straight-Medial Axis Transformation (S-MAT) is proposed in (Eppstein and Erickson, 1999), and it results to be a good representation of natural human behaviour, though, it may not represent accessibility within buildings accurately (Mortari, 2013).

Generally, the granularity of the semantic models is kept relatively coarse. The smallest identifiable space is a room, which is approximately regarded as a node within the derived network. Detailed partition of a room is rarely considered. Zlatanova et al. (2013) propose a framework for space subdivision considering four components: agents, activities, resources and modifiers. Kruminaite and Zlatanova (2014) present a model to identify functional spaces around objects and obstacles, which can be used for localisation and navigation. Still the question remains whether to consider a detailed partition (regular or irregular) of a space considering all obstacles. Regular partition of indoor spaces has been largely employed in raster-based navigation, which is the basis for robot navigation (Afyouni et al., 2012). Delaunay triangulation has been also commonly applied for quick subdivision of large spaces and thus increases the number of the nodes in the derived network. The network for navigation is then obtained from the centre points of the triangles. However, the obtained path on such network is quite unrealistic and requires improvements (Mortari et al., 2014; Kruminaite and Zlatanova, 2014).

This paper proposes an indoor navigation approach that considers obstacles of different shapes, subdivides the remaining navigable space and creates a network, which provides a realistic navigation. The remainder of this paper is organised as follows: Section 2 presents indoor information processing. Section 3 discusses the establishment of the indoor network. Section 4 presents the implementation and experiments and Section 5 concludes this paper and discusses the future work.

\section{INDOOR INFORMATION PROCESSING}

The information of common 2D floor plan drawings cannot be directly used for navigation, neither the information available as a digital plan or stored in databases (Wu, 2015). Therefore, available indoor spatial information has to be processed to create indoor network that can be used for path computation. Indoor network is a graph representing connectivity of indoor spaces, which concisely describes the surrounding environment and contains enough semantic, geometrical and topological information. In general, indoor space contains public area, accessible and inaccessible area. These areas are usually separated by obstructing structures such as walls. Additionally there are many objects inside the rooms, such as tables and chairs, which also act as obstacles. They divide the space into specific functional areas for different purposes (Xu, 2009; Kruminaite and Zlatanova, 2014). When constructing indoor network, we must solve the problem of representation of obstacles and create a path that avoids them (Liu and Zlatanova, 2015).

Generally, indoor objects can be described as points, lines and polygons in $2 \mathrm{D}$. We describe indoor objects with points and lines. Doors are represented by their midpoints. Walls are represented as single lines connected by their endpoints. Indoor objects are represented as lines, triangles and polygons according to their size and location in the room.

After the initial 2D floor plan is redrawn and all the obstacles are correctly presented, the question is how to extract the network that will be used for path computation. Therefore, it's necessary to deal effectively with the remaining free space, which needs to be subdivided. In this paper, we use Delaunay triangulation to create a mesh (Du et al., 2015; Yu, 2013). The 
subdivision method follows a "maximum of the minimum angle" and an "empty circle" criteria. Delaunay triangulation can satisfy optimally both global and local levels based on these two criteria. Furthermore, it's adaptable for cases in which regional boundary and internal medium boundary are irregular and the distribution of field phenomena changes as well (Xu, 2009). Therefore Delaunay triangulation is selected for indoor space subdivision in this paper.

\section{ESTABLISHMENT OF INDOOR NETWORK}

\subsection{Indoor Space Subdivision}

The indoor network is depended on the configuration and the size of the spaces in a building. If a room is empty and relatively small, a subdivision is most commonly not needed and the shortest path is the straight line connecting starting point and target point in the room. However, large areas such as sitting room, shopping centre hall, etc. do require space subdivision. Such spaces contain many obstacles. In this case, the path must avoid obstacles and yet be the shortest ( $\mathrm{Lu}$ et al., 2014).

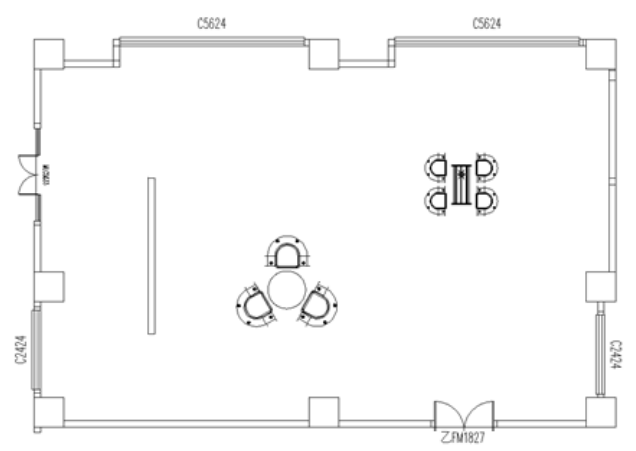

Figure 1. Layout of Room A

Figure 1 shows the layout of Room A $(6 \mathrm{~m} * 4 \mathrm{~m})$. Following the simplification approach, as described above, we obtain the new representation needed for the path computation. The walls, including indoor walls, are expressed as straight lines, the door's midpoint represents the door, and indoor objects are abstracted as lines, triangles and polygons according to their shapes (Figure 2).

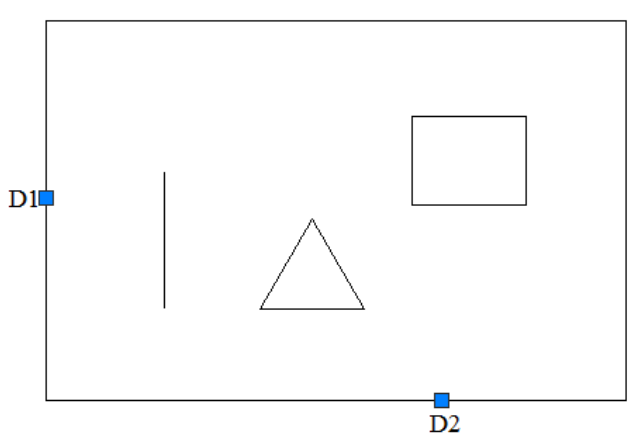

Figure 2. Simplified plan of Room A

After the 2D floor plan is simplified and re-organised, the indoor space excluding obstacles needs to be subdivided. Such a large indoor space is divided into multiple small areas, using constrained Triangulated Irregular Network (TIN) (Zheng et al., 2012). A threshold needs to be set up in the function of constructing TIN (pTRI $=$ Create_Tri () ), because when all sides are smaller than the threshold value, a TIN of high quality can be constructed such that all edges of obstacles and walls appear in it. There are total of 22 triangles for the room represented in Figure 2. The redundant triangles, which exist within the obstacles have to be deleted to get the final triangulation. After this step, 19 triangles are left (Figure 3).

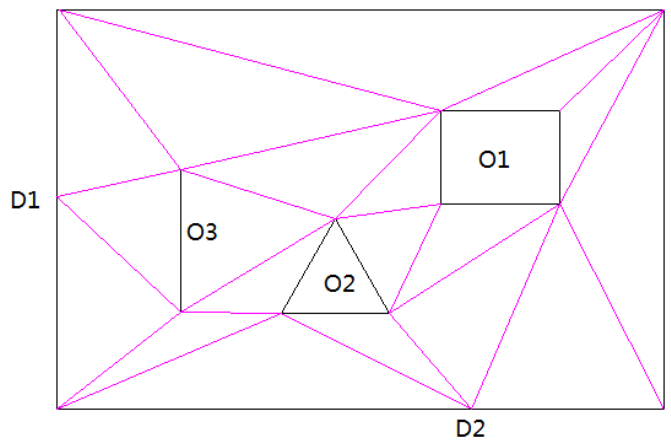

Figure 3. Regional subdivision graph

\subsection{Construction of the Network}

The indoor space is refined after the subdivision and the network can be created. Each triangle is represented by a point which becomes a node in the network for navigation. In some cases, a certain feature point (table, chair, bed, plant) can be found to represent the area, wherever inside the triangle. In other words, when person moves from one area to another, it can be regarded as point-to-point movement (from the 'table' to 
the 'bed' and to the 'door'). Lines connecting these feature points indicate the edges of the navigation network (Xu, 2009). The selection of feature points in our case is easy and straightforward. The centre of gravity of a triangle is chosen as a feature point. The nodes are obtained by traversing all triangles (19) shown in Figure 4.

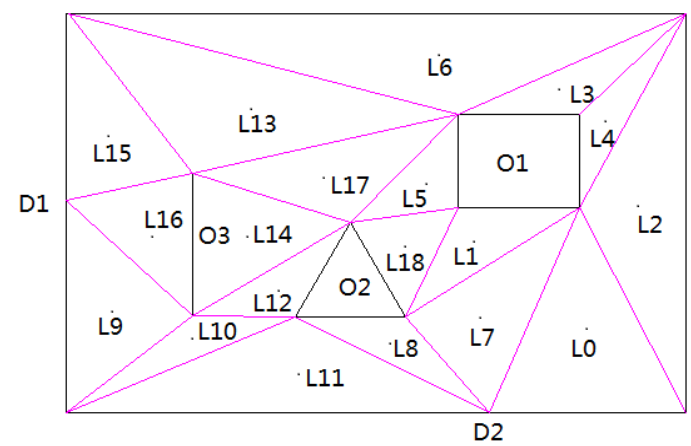

Figure 4. Area feature points

\subsection{Establishment and Optimization of Path}

To be able to create the network it should be decided how to connect the nodes in such a way to avoid the commonly used connection between neighbouring triangles, which results in zigzagging paths. Therefore we chose to connect all the nodes excluding edges that cross the obstacles. In above example, there are 210 lines connecting nodes without taking obstacles into account. We eliminated the edges that cross obstacles by checking if the obstacle lines intersect with the lines of network nodes. If intersection is detected, the line should be ignored. The intersection of two lines is performed by checking if two points are on either side of a line (Figure 5).

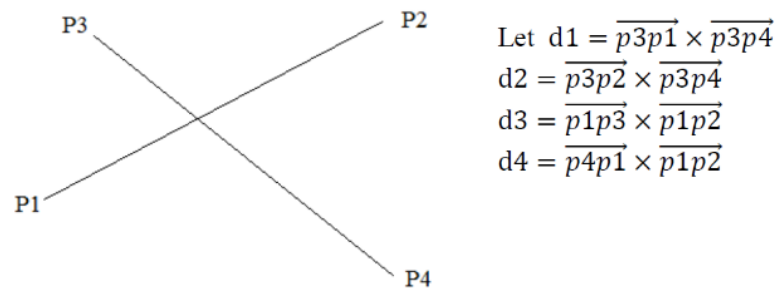

Figure 5. Line segment intersection

As long as $\mathrm{d} 1 * \mathrm{~d} 2<0$ and $\mathrm{d} 3 * \mathrm{~d} 4<0$, line $\mathrm{p} 1 \mathrm{p} 2$ inevitably intersects with line p3p4. However, if the result is zero, it's necessary to determine whether the corresponding point is on the line.

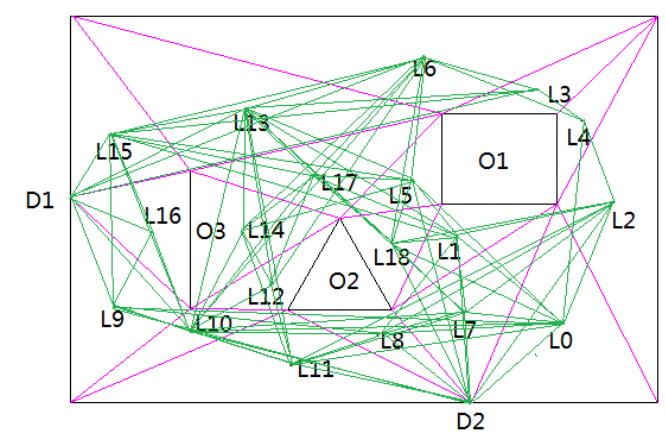

Figure 6. Resulting network for navigation (green lines)

Lines of obstacles and lines of network nodes are compared one by one based on above conditions. Following this approach 85 lines are left after eliminating intersection lines by traversing all lines (Figure 6).

\section{IMPLEMENTATION AND EXPERIMENTS}

The approach mentioned above for processing the $2 \mathrm{D}$ floor plan and creating navigation network has been implemented and tested on several 2D floor plans. After the network is created, the shortest path is computed using Dijkstra algorithm, which is widely used (Zheng et al., 2012; Liu, 2013; Xu, 2010) and faster compared to A* algorithm (Chen and Dong, 2010; Yang, 2014; Wang, 2012). Dijkstra algorithm is now regarded as the most classical algorithm for finding the shortest path, which can be applied to all cases as long as the weights are positive.

As shown in Figure 2, we select two doors (D1 and D2) respectively as start point and end point for the path computation. Figure 7 shows the shortest path based on Dijkstra algorithm between two doors.

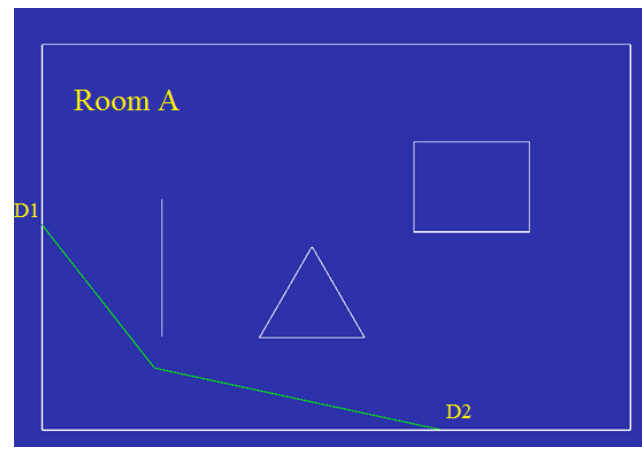

Figure 7. The shortest path between D1 and D2 in Room A (green) 
Figure 7 illustrates that there is a turning point starting from one door and the target door can be reached by changing direction at this point and walking some distance. The turning point is one of feature points of previous subdivided areas in Figure 4. In this case it can be indicated as 'the corner of the wall'.

The proposed approach, in contrast to 'door-to-door' approach (Liu and Zlatanova, 2013), consider that the obstacles are simplified concave objects and therefore intermediate points of a path between a source and a target are only the concave corners. Furthermore, the amount of space required for storing nodes and edges, the number of possible combinations is quadratic (Mortari, 2013). The proposed approach can deal with concave spaces with irregular obstacles and the number of network edges can be controlled to avoid crossing with the obstacles.

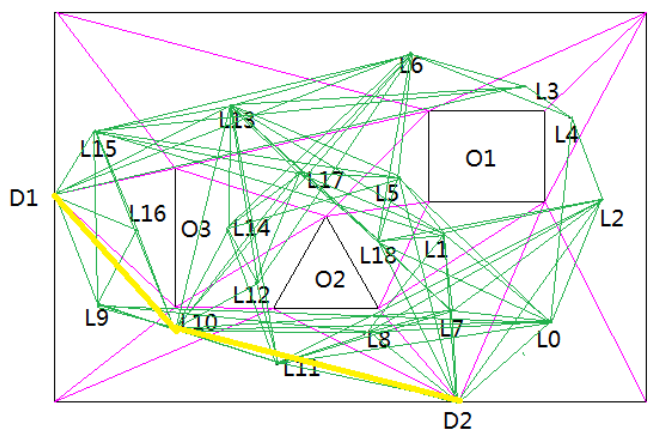

(a) Proposed approach resulting network

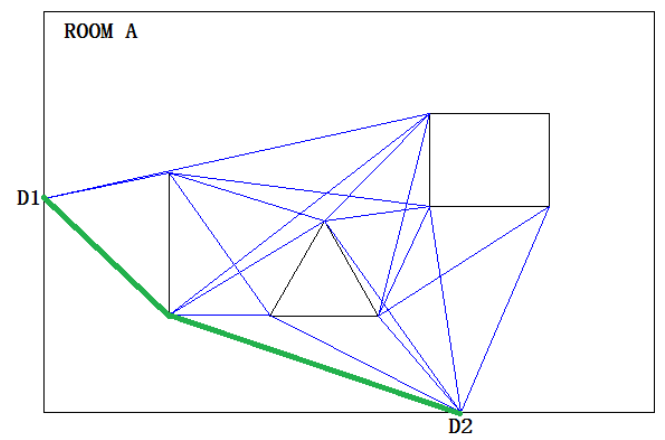

(b) Door-to-Door approach resulting network

Figure 8. Comparison between the proposed and Door-to-Door

The following example illustrates the approach in case of irregular obstacles. In Room B (6 m* 4 m), irregular obstacles are described as convex polygons or concave polygons according to its shape (Figure 9). Figure 10 shows resulting network in Room B and the shortest path between doors D1 and D2.

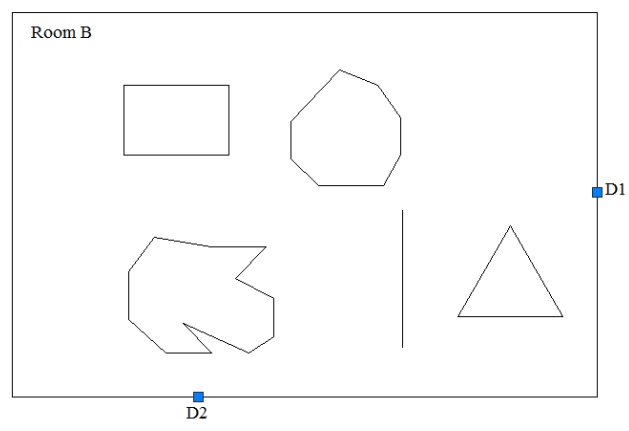

Figure 9. Simplified plan of Room B

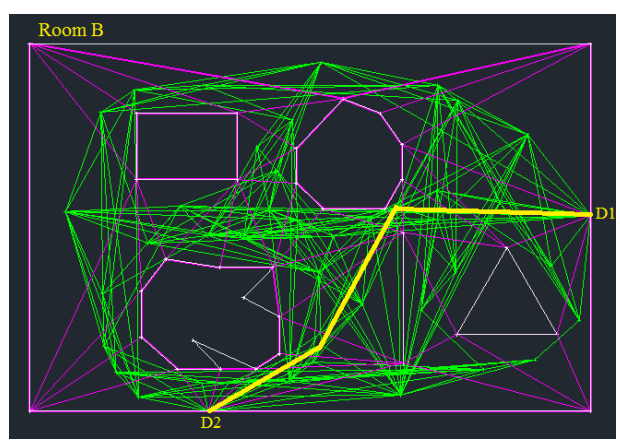

Figure 10. Resulting network for navigation (green) and the shortest path (bold yellow)

Another more elaborated space is illustrated below, i.e. Room $\mathrm{C}$ with the size of $9.8 \mathrm{~m} * 7.0 \mathrm{~m}$ (Figure 11). The indoor layout as redrawn according to our implication rules is shown in Figure 12.

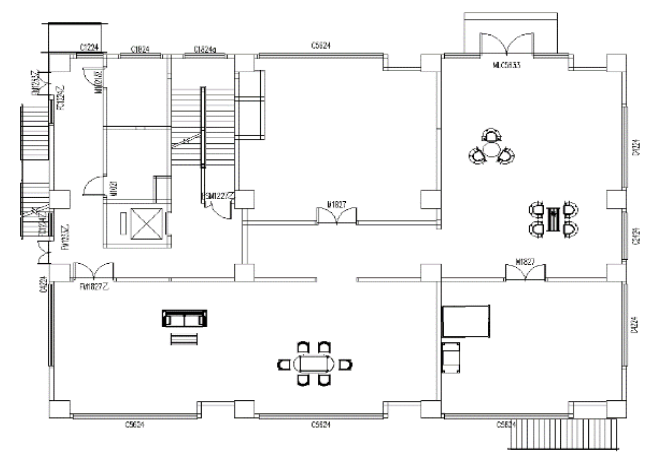

Figure 11. Layout of Room C 


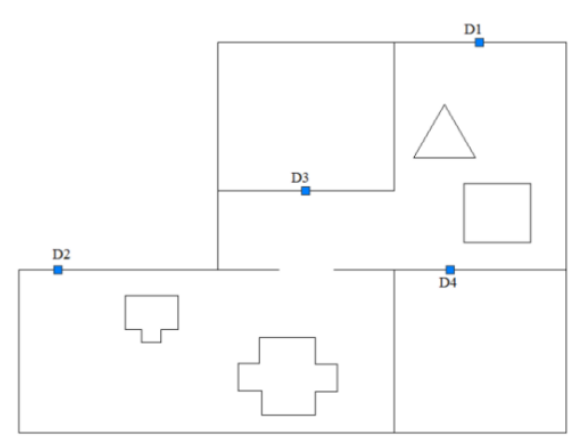

Figure 12. Simplified plan of Room C

Doors D1 and D2 are set respectively as start point and end point, and resulting network for navigation is shown in Figure 13. The approach searches the shortest route connecting the two points, which is marked in bold yellow lines. Inside red box are some features points outside Room $\mathrm{C}$, which we can ignore. And detail in blue box is magnified, which shows that network doesn't intersect with obstacles' edges.
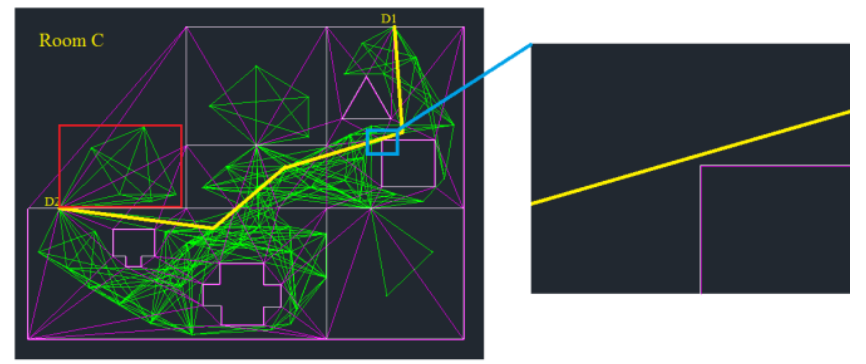

Figure 13. Resulting network for navigation (green) and the shortest path (bold yellow)

Now we take a more elaborated and larger space for an example, the first floor of the No. 1 Teaching Building of Beijing University of Civil Engineering and Architecture in Daxing campus with the size of $46 \mathrm{~m} * 13 \mathrm{~m}$ (Figure 14).

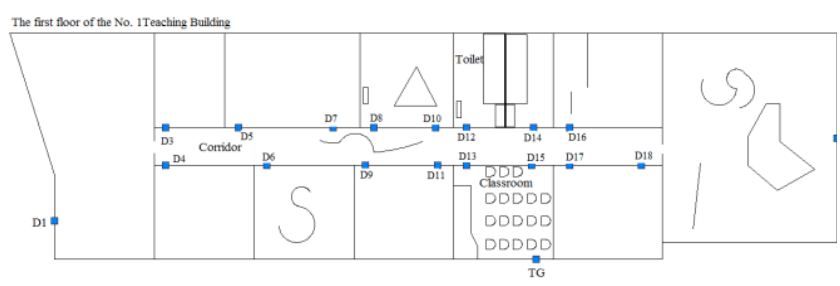

Figure 14. Simplified plan

Doors D1 and D2 are set respectively as source and target.

Figure 15 shows resulting network as well as the shortest path, and features points outside simplified plan's boundary are ignored.

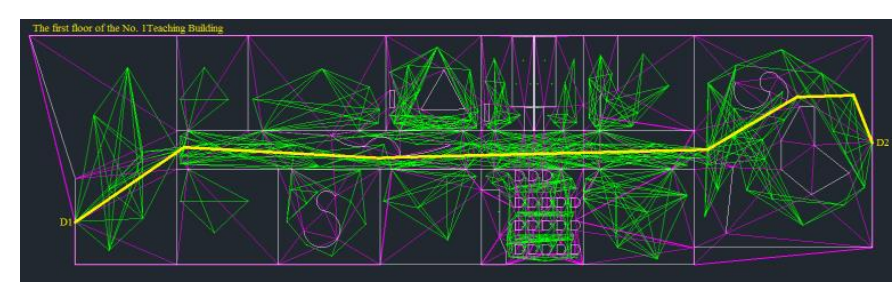

Figure 15. Resulting network for navigation (green) and the shortest path (bold yellow)

This approach provides an optimal non-circuitous routes and bypasses the obstacles validly, while S-MAT may create distorted paths for large arbitrary shaped spaces. For instance, it may create weird networks for corridors (Mortari, 2013).

Next, Door D1 and position TG are set respectively as source and target. The test is used to demonstrate the approach more convincingly. Doors, as well as, the centres of gravity of triangles are chosen as feature points in this case. We can walk around the desks in the classroom according to the computed shortest path (Figure 16).

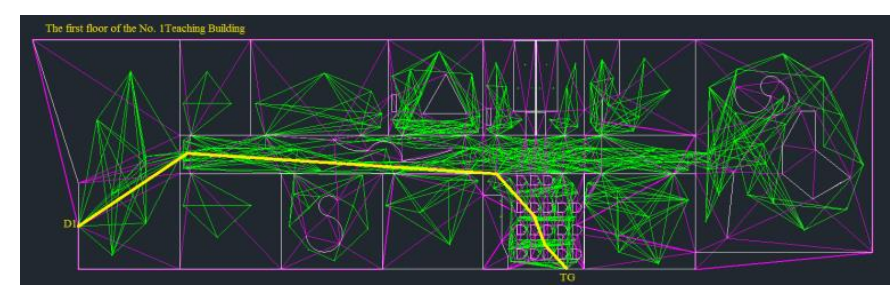

Figure 16. Resulting network for navigation (green) and the shortest path (bold yellow)

The approach is proved to be very promising for indoor navigation, according to verification results of above examples. The approach is able to deal with all kinds of obstacles, regardless of whether they are regular or not. Additionally, this approach shows that zigzagging routes can be avoided when Delaunay triangulation is applied for subdivision of navigable space.

\section{CONCLUSION}

In this paper, we address the subdivision of free space inside buildings. Firstly we analyse indoor spatial structure and simplify 2D floor plans to automatically obtain a navigation network. The subdivision of the space is based on Delaunay triangulation. We abstract each triangle with a feature point, which is a node in the network. We construct the network, 
which means connect all the nodes and remove the edges that cross obstacles.

As mentioned above, S-MAT and 'door-to-door' approaches have some limitations besides valuable merits. Detecting large arbitrary shaped spaces is evidently the weakness of S-MAT. The approach in this paper helps to overcome the problem of distortion of the path towards the middle of the open space especially in presence of obstacles. Moreover, by linking the feature points with edges, which do not cross obstacles, the network encompasses the positive aspects of a "door-to-door" navigation between intervisible locations. It can be used for real time computation. It takes a little time to run the approach on the small examples presented above. It should be investigated what strategy should be applied for a huge complexes. An approach would be to create the network only per room and construct it on the fly when needed (Liu and Zlatanova, 2013).

The approach in this paper can only be used for 2D floor plans and path-finding. The simplification of $2 \mathrm{D}$ floor plan is currently done manually. Further research is need for fully automated simplification. In the future work, we will investigated description and processing of 3D indoor models, specifically semantically-rich building models such as CityGML LoD4 and IFC.

\section{ACKNOWLEDGEMENT}

This work is supported by Young Talents Training Programme of Beijing (No. 21147514067), the Dutch project M4S 13742 Smart 3D indoor models to support crisis management in large public buildings (www.sims3d.net), State Key Development Program of Basic Research of China (No. 2012CB725301), the National Natural Science Foundation of China (No. 40801159) and Key Laboratory for Urban Geomatics of National Administration of Surveying, Mapping and Geoinformation (No. 20111215N) .

\section{REFERENCES}

Afyouni, I., Ray, C. and Claramunt, C., 2012. Spatial models for context-aware indoor navigation systems: A survey, Journal of Spatial Information Science, Number 4 (2012), pp. $85-123$.

Chen, S. Q. and Dong, L. F., 2010. Applied analysis of Dijkstra Algorithm and A-star Algorithm in the Intelligent Guiding.
Journal of Chongqing Institute of Technology (Science and Technology), 12 (6), pp. 159-161.

De Berg, M., Van Kreveld, M., Overmars, M., and Schwarzkopf, O. C., 2000. Chapter 15: Visibility Graphs. Computational geometry (2nd ed.). Springer Berlin Heidelberg.

Du, H. K., Feng, D. S. and Tang, J. S., 2015. GPR simulation by finite element method of unstructured grid based on Delaunay triangulation. Journal of Central South University (Science and Technology), 46 (4), pp. 1326-1334.

Eppstein, D. and Erickson, J., 1999. Raising Roofs, Crashing Cycles \& Playing Pool: Applications of a Data Structure for Finding Pairwise Interactions. In: 14th Annual ACM Symposium on Computational Geometry, Minneapolis, USA, pp. 58-67.

Goetz, M. and Zipf, A., 2011. Formal definition of a user-adaptive and length-optimal routing graph for complex indoor environments. Geo-Spatial Information Science, 14 (2), pp. 119- 128 .

Kruminaite, M. and Zlatanova, S., 2014. Indoor Space Subdivision for Indoor Navigation, ISA'14, Proceedings of the Six ACM SIGSPATIAL International Workshop on Indoor Spatial Awareness, pp. 25-31.

Li, D. D. and Lee, D. L., 2008. A topology-based semantic location model for indoor applications. In Proceedings of the 16th ACM SIGSPATIAL International Conference on Advances in Geographic Information Systems, pp. 9. ACM.

Liu, Y. W., 2013. Research on the optimal path algorithm in Intelligent Transportation System. Master Thesis, Kunming University of Science and Technology.

Liu, L. and Zlatanova, S., 2015. An approach for indoor path computation among obstacles that consider user dimension, ISPRS International Journal of Geo-Information, 4(4) pp. 2821-2841.

Liu, L. and Zlatanova, S., 2013. A two-level path-finding for indoor navigation, In: S. Zlatanova, R. Peters, A. Dilo and H. Scholten (Eds.); Intelligent systems for crisis response, LNG\&C, Springer, Heidelberg, New York, Dordrecht,London, pp. 31-42. 
Liu, L. and Zlatanova, S., 2011. A "door-to-door" path-finding approach for indoor navigation, In: Altan, Backhause, Boccardo \& Zlatanova (Eds.), International Archives ISPRS XXXVIII, 7th Gi4DM, 3-7 May, Anltalya, Turkey, pp. 3-8.

Lu, W., Wei, F. Y., Zhang, S. and Suo, R. Y., 2014. Research on a method of Constructing the Indoor Road Network Model. Journal of Navigation and Positioning, 2 (4), pp. 63-67.

Mortari, F., 2013. Automatic extraction of improved geometrical network model from CityGML for indoor navigation. Master Thesis, University of L'Aquila.

Mortari, F., Zlatanova, S., Liu, L. and Clementini, E., 2014. "Improved Geometric Network Model" (IGNM): a novel approach for deriving Connectivity Graphs for Indoor Navigation, In: A. Karpik and V. Seredovich (Eds.), ISPRS Ann. Photogramm. Remote Sens. Spatial Inf. Sci, Vol. II-4, pp. 4551.

Wang, D. J., 2012. Indoor mobile robot path planning based on the improved A* algorithm. Journal of Tsinghua University (Science and Technology), 52 (8), pp. 1085-1089.

Worboys, M., 2011. Modeling indoor space. In Proceedings of the 3rd ACM SIGSPATIAL International Workshop on Indoor Spatial Awareness. pp. 1-6. ACM.

$\mathrm{Wu}, \mathrm{H}, 2015$. Integration of $2 \mathrm{D}$ architectural floor plans into Indoor Open street map for reconstructing $3 D$ building models. Master Thesis, Delft University of Technology, http://repository.tudelft.nl/view/ir/uuid\%3A26a4c6b8-8ea0-424 6-b30e-5c83a28cccb4.

$\mathrm{Xu}$, J., 2009. Research on path planning method of the indoor mobile navigation system. Master Thesis, Changchun University of Science and Technology.
Xu, J. J., 2010. Optimal Path Algorithm Research In Vehicle Navigation System. Master Thesis, Chang' an University.

Yang, Y. T., 2014. Obstacle avoidance application simulation based on $A^{*}$ algorithm. Master Thesis, Zhengzhou University.

Yu, Y. L., 2013. Research on the Problems Related to Delaunay Triangulation and Voronoi Diagram in GIS. Master Thesis, Anhui Normal University.

Zhao, X. Y. and Liu, H. Q., 2010. An indoor navigation algorithm based on concept lattice. Public science and technology, Number 4 (2010), pp. 40-42.

Zheng, M. X., Wang, Y. B. and Ma, X. X., 2012. An Effectively Combined Algorithm of Generating TIN Based on the Quad-Tree Structured Index. Geography and Geo-Information Science, 28 (2), pp. 20-23+59.

Zheng, Y. J., Hou, K. H., Liao, W. Z. and Yang, L., 2012. The Shortest Path Algorithm Based on Petri Net. In Proceedings of 2012 IEEE 19th International Conference on Industrial Engineering and Engineering Management (IE \& EM 2012), Chang Sha, China, pp. 4.

Zlatanova, S., Liu, L. and Sithole, G., 2013. A Conceptual Framework of Space Subdivision for Indoor Navigation. ISA '13, Proceedings of the Fifth ACM SIGSPATIAL International Workshop on Indoor Spatial Awareness, ACM New York, NY, USA. pp. 44-48.

Zlatanova, S., Liu, L., Sithole, G., Zhao, J. and Mortari, F., 2014. Space subdivision for indoor applications, GISt Report, nr. 66, Delft, pp. 50. 